\title{
Training the trainees: COVID-19 and rheumatologists of the future
}

\author{
Authors: Hannah Jethwa ${ }^{A}$ and Sonya Abraham ${ }^{B}$
}

KEYWORDS: training, junior doctors, general medicine, rheumatology, future of medicine

DOI: 10.7861/clinmed.2020-0907

The outbreak of SARS-CoV-2 has resulted in wide-ranging turmoil at an international level. During the peak of the pandemic, healthcare services in countries worst hit by COVID-19 were required to drastically reconfigure models of care delivery in order to optimise access to both resources and a workforce available to aid in the care of critically unwell patients. Many elective and nonurgent services were placed on hold during this time and doctors from a multitude of specialties were redeployed in order to support general medical care needs. Although the recent relative reduction in new cases of COVID-19 has allowed for specialist services, such as outpatient clinics, to be reinstated, the slow reintroduction of normality within the healthcare sector is likely to have longlasting implications on specialist clinical training. Consideration is therefore required into measures that need to be implemented to ensure ongoing competence and confidence among trainees, especially for specialties such as rheumatology, where concerns regarding immunosuppression are likely to impact models of elective service delivery for many months or years to come.

With the advent of new and advanced technologies, the concept of telemedicine has evolved, however, its implementation had, until recently, been relatively sparse around the globe. The onset of the COVID-19 pandemic, however, prompted a rapid and widespread uptake in remote consultations and patient self-monitoring platforms and many rheumatology services internationally have utilised such modalities over recent months to minimise the need for face-to-face visits with patients on immunosuppressive agents. ${ }^{1-4}$ Although a handful of rheumatology centres have published qualitative data on local experiences with the use of virtual consultations and remote patient monitoring during this time, further qualitative and quantitative research is required at both national and international levels to demonstrate both patient and healthcare provider satisfaction as well as the clinical effectiveness of telemedicine in our cohort of patients. ${ }^{2,3,5}$ Clinical consultations

Authors: ${ }^{\text {A }}$ specialist registrar in rheumatology and general internal medicine, Northwick Park Hospital, London, UK; ${ }^{B}$ consultant research fellow and rheumatologist, Imperial College London, London, UK carried out via telephone or video calls are likely to be more robust when delivered by healthcare providers who have followed up these patients over a period of time and therefore are able to highlight pertinent aspects of their care more confidently without the need for a face-to-face visit. For trainees, however, the inability to carry out a physical examination, lack of specific virtual training and difficulty in picking up physical cues from patients can often lead to apprehension and a lack of confidence in implementing virtual reviews, especially for patients with complex needs. ${ }^{6}$

In the USA, where telemedicine has been an expanding field over the past few decades, diverse approaches have been undertaken by various universities to develop such competencies in medical students to allow for accelerating growth in this domain. ${ }^{7}$ In other countries, however, such training has been limited to date. Although online training resources are available to highlight ways to conduct effective remote consultations, given the probable longevity of this pandemic, such training should be included in future undergraduate and postgraduate training curricula, and specialty-specific training for such consultations would also aid with standardisation and harmonisation of the way that trainees carry out specialist virtual reviews during this time. ${ }^{8}$

Given that virtual consultations are likely to continue for the foreseeable future, now is the time for national training bodies to re-evaluate how to meet specialty-specific training needs for the present and future, not only to ensure a standard of clinical safety and effective care for patients but also to enable adequate advancement in clinical abilities to warrant training progression as well as avoid trainees from becoming unskilled or de-skilled in certain areas. A large aspect of early training in rheumatology consists of clinically differentiating between normal and abnormal physical examinations. For example, in order to gain confidence at diagnosing joint pathology, one must have experience in examining normal joints; furthermore, this experience of performing regular joint examinations enables confidence in later differentiating between more subtle pathologies, such as synovitis from soft tissue swelling. Although much of the academic aspects of teaching can be delivered virtually during this time, development of such clinical skills remains a significant challenge, especially for junior trainees; additionally, even for experienced trainees, the potential for limited face-to-face consultations for a prolonged period of time may result in them becoming de-skilled with regards to physical examinations. Procedural competence is also a significant challenge during this time as the majority of centres are only performing intra-articular joint injections in a subset of urgent cases and are likely to resort to more conservative 
measures, such as prescribing a short course of low-dose glucocorticoids for flares of joint inflammation, rather than asking patients to attend outpatient services.

With these challenges in mind, it is vital that such aspects of rheumatology training are reviewed and restructured to reflect these unprecedented complexities and enable confidence and competence of trainees during this time. Furthermore, deliberation is needed regarding aspects of clinical training post-pandemic, when the frequency of face-to-face consultations increases. One solution to ensure clinical and procedural competencies among trainees and specialists may be implementation of various support modalities, including simulation sessions, similar to the UK-based Supported Return to Training (SuppoRTT) scheme, which is currently available for trainees returning to clinical roles following breaks in training (such as for parental leave or research posts). ${ }^{9}$ It is also imperative that training curricula include new aspects of clinical management that are relevant during this era, including new core competencies for immunology, vaccinology and clinical pharmacology, both generally and related to rheumatic disease. Given the potential of multisystem involvement of COVID-19 and the chance of further specialty redeployment in the future, curricula and training programmes should emphasise the importance of a strong foundation of general medical knowledge to enable adaptability of clinical roles. This review of training is not only key for allowing smooth and efficient service delivery over the next few months and years but it is also crucial to ensure patient safety in years to come.

\section{References}

1 Jethwa H, Abraham S. Should we be using the Covid-19 outbreak to prompt us to transform our rheumatology service delivery in the technology age? Rheumatol 2020;59:1469-71.
2 Zhang Y, Wang J, Zhao L, Xiao J, Shi Z. Online management of rheumatoid arthritis during COVID-19 pandemic. Ann Rheum Dis 2020;annrheumdis-2020-217548 [Epub ahead of print].

3 Lopez-Medina C, Escudero A, Collantes-Estevez E. COVID-19 pandemic: an opportunity to assess the utility of telemedicine in patients with rheumatic diseases. Ann Rheum Dis 2020;annrheumdis-2020-218008 [Epub ahead of print].

4 Kirby $T$. Rheumatologists rapidly adjust patient care during COVID-19 pandemic. Lancet Rheumatol 2020;2:E258.

5 Bozzalla Cassione E, Zanframundo G, Biglia A et al. COVID-19 infection in a northern-Italian cohort of systemic lupus erythematosus assessed by telemedicine. Ann Rheum Dis 2020:annrheumdis-2020-217717 [Epub ahead of print].

6 Chaudhry U, Ibison J, Harris T et al. Experiences of GP trainees in undertaking telephone consultations: a mixed-methods study. BJGP Open 2020;4:bjgpopen20X101008.

7 Waseh S, Dicker AP. Telemedicine training in undergraduate medical education: mixed-methods review. JMIR Med Educ 2019;5: e12515.

8 Hammerton C, Royal College of Physicians. Effective remote consultations. London: RCP, 2020. www.rcplondon.ac.uk/educationpractice/courses/effective-remote-consultations [Accessed 06 August 2020].

9 England Health Education. Supported Return to Training. HEE. www.hee.nhs.uk/our-work/supporting-doctors-returningtraining-after-time-out [Accessed 06 August 2020].

Address for correspondence: Dr Hannah Jethwa, Department of Rheumatology, Northwick Park Hospital, Watford Road, Harrow HA1 3U], UK.

Email: hannahjethwa@nhs.net 